\title{
TRANSITION CROSSING IMPROVEMENTS
}

\author{
M. Church
}

25 April 1991

\section{Motivation}

During the accelerator studies period of $12 / 90$ - 1/91 efforts were made to improve the efficiency of crossing transition in the Accumulator. E760 wishes to measure the product of the branching ratios for

$$
\bar{p} p \longrightarrow \eta_{c} \longrightarrow \gamma \gamma
$$

and the mass and width of the $\eta_{c}$. Since resonance production of the $\eta_{c}$ requires a beam momentum of $3.67 \mathrm{Gev} / \mathrm{c}$ which is below the Accumulator design transition momentum, and since the above process is rare, it is required to cross transition effciently $(>90 \%$ ) with $30 \mathrm{~mA}$ of beam. In the past, transition crossing efficiency has been intensity-dependent, with never more than $10 \mathrm{~mA}$ getting through transition. At $8 \mathrm{~mA}$ or less, transition crossing efficiency was typically $100 \%$. The beam loss is due to a large longitudinal beam blow-up which has been observed many times during transition crossing (see Fig. 1).

\section{Transition Crossing Procedure}

Beam is decelerated to a momentum of $5.2 \mathrm{Gev} / \mathrm{c}$ by slowly ramping down all Accumulator magnets and $\mathrm{RF}(\mathrm{H}=2)$. Starting at approximately $6.5 \mathrm{Gev} / \mathrm{c}$ the quadrupole strengths are ramped so as to distort the Accumulator lattice so that $\gamma_{T}$ drops to 5.0 . At $5.2 \mathrm{Gev} / \mathrm{c}$ the beam is debunched and the quadrupoles alone are ramped so that $\gamma_{T}$ rises to 6.2 . This is traditionally called a " $\gamma_{T}$ jump." The beam is then rebunched and decelerated as before to lower momenta. The quadrupole strengths are ramped such that the design lattice is restored and $\gamma_{T}$ is restored to 5.5. Fig. 2 shows $\gamma_{T}$ as a function of beam momentum. Beam is stochastically cooled in all 3 dimensions after transition crossing but not before (during normal operation). Just before transition crossing $\eta=-.0075$, and just after transition crossing $\eta=.0058$. The transition crossing part of the ramp consists of 26 separate current steps with $1 / 2$ second pauses between; although the actual transition crossing occurs at a single step-step 15. The changes in the bus currents are as follows:
A:QT
147.3 amps to 125.6 amps
$-15.9 \%$
A:LQ
692.5 amps to 758.1 amps
$+9.0 \%$
$A: Q D$
137.7 amps to 141.5 amps
$+2.7 \%$ 
A:QF $\quad 137.5$ amps to 139.5 amps $\quad+1.4 \%$

During transition crossing the tunes are kept constant at $Q_{h}=.615 \pm .002$ and $Q_{v}=.612 \pm .002$. $\gamma_{T}$, which depends mostly on LQ, is changed approximately linearly with time (see Fig. 3). Since the beam is not centered in the quadrupoles, it is mis-steered by the quadrupoles and the apertures are reduced substantially during transition crossing:

horizontal: $10.2 \pi \mathrm{mm}$-mrad before transition, $7.0 \pi \mathrm{mm}-\mathrm{mrad}$ after transition vertical: $10.1 \pi \mathrm{mm}$-mrad before transition, $6.3 \pi \mathrm{mm}-\mathrm{mrad}$ after transition.

However, transverse emittances remain adequately small during transition crossing so as not to cause beam loss.

\section{Studies}

A Monte Carlo simulation ${ }^{1}$ of transition crossing indicated that the longitudinal beam blow-up was relatively slow ( $\sim 1 / 2 \mathrm{sec}$ ), and that it was primarily caused by self-bunching of the beam via the longitudinal impedance $(\sim 50 \mathrm{k} \Omega)$ of the ARF1 cavities. The frequency spectrum from the resistive wall monitor (a broadband pickup) had always shown a strong coherent peak at $53 \mathrm{Mhz}$, which is the resonant frequency of the ARF1 cavities.

For these reasons it was decided to implement the following changes:

1) cross transition more quickly;

2) short the ARF1 cavities during transition crossing so their shunt impedance is reduced to $\sim 0$;

3) short the ARF3 cavity during transition crossing, increasing its resonant frequency to $14 \mathrm{Mhz}(Z \rightarrow \sim 100 \Omega)$.

Crossing transition more quickly required that steps $12-18$ in the quadrupole current ramps be decreased from $1 / 2 \mathrm{sec}$ to $1 / 12 \mathrm{sec}$ each. Since the response time of the QT power supply is slow, at these setting rates the actual bus current does not immediately correspond to the current setting. Therefore the correct current ramp for this device had to be determined empirically by trial and error. For the other devices, the time scale could simply be reduced by a factor of 6 for the relevant steps. Fig. 4 shows the QT and QDF bus currents as a function of time for the slow ramp (1/2 sec per step) and the fast ramp (1/12 sec per step for steps 12-18).

The ARF1 short consists of a metal cylindrical sleeve which is motor-driven across the RF gap. The ARF3 short is simply a closed switch across the gap.

Tests with $7 \mathrm{~mA}$ of forward protons showed that, by itself, shorting ARF1 eliminated the coherent spike at $53 \mathrm{Mhz}$ during transition crossing, as did the faster ramp by itself. Fig. 5 shows the effect of both the faster ramp and shorting ARF1. The momentum blow-up was also reduced by these modifications. Shorting ARF3, in addition, seemed to make no difference, however the region around $1.2 \mathrm{Mhz}$ was not studied carefully. Transition crossing was later tried with $7 \mathrm{~mA}$ of $\bar{p}$. Fig. 6 shows the longitudinal Schottky spectrum before and after transition crossing. The ratio

$$
\Delta p_{\text {below }} / \Delta p_{\text {above }}=\left(\Delta f_{\text {below }} \eta_{\text {above }}\right) /\left(\Delta f_{\text {above }} \eta_{\text {below }}\right)=.8
$$

${ }^{1} \mathrm{Q}$. Wang, thesis to be submitted shortly 
indicates there is no momentum blow-up while crossing transition. Transition crossing was not attempted with $30 \mathrm{~mA}$ of forward protons because of possible radiation damage to the $\mathrm{E} 760 \mathrm{~Pb}-\mathrm{G}$ calorimeter.

\section{Conclusions}

For small beam currents $(\leq 7 \mathrm{~mA})$ the two modifications-shorting ARF1 and crossing transition more rapidly-eliminate the longitudinal beam blow-up, and the coherent self-bunching of the beam during transition crossing. It is not clear if shorting ARF3 contributes. It remains to be seen if these techniques are effective at 30 $\mathrm{mA}$ beam current. All 3 modifications have been integrated into the standard E760 deceleration procedures. 
点

- Console locatian Os, mCRlio

blaw-up at transition

$3-30 L-1990 \quad 11: 01$

7 MA $\bar{P}$

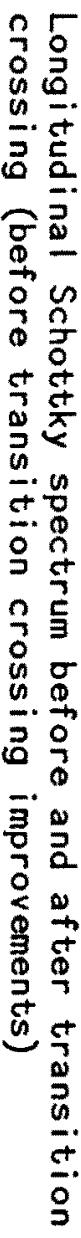

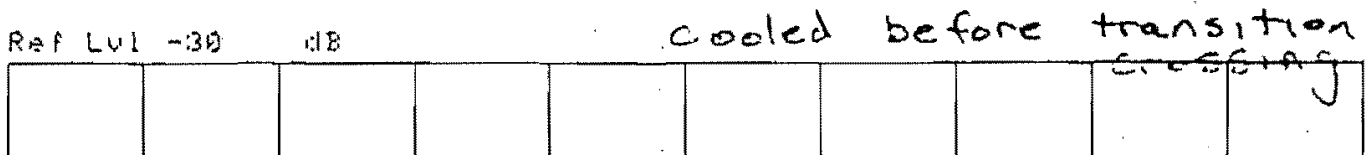

$07 / 03 / 30$ 0315

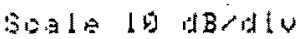
$A \tan \theta \quad d B$ Sup 3 … son VIO BH $100 \mathrm{~Hz}$ $R=B H \quad 100 \mathrm{~Hz}$
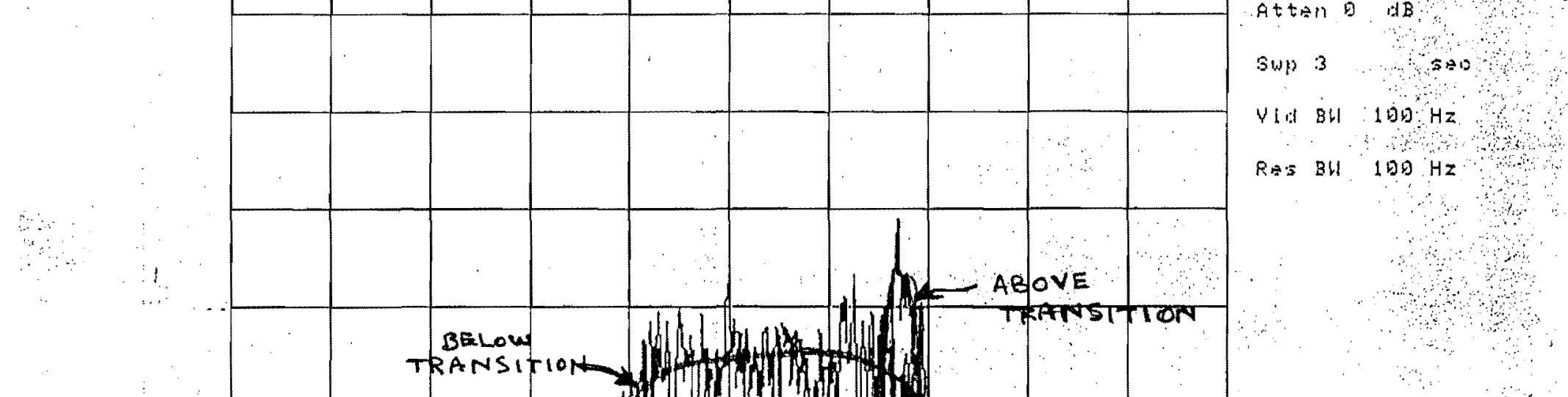

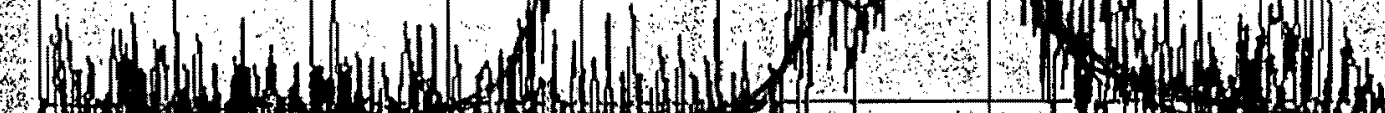
of

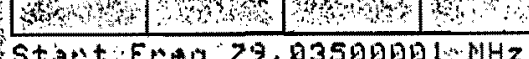

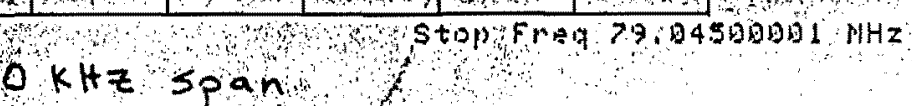

$0 K H z \operatorname{span} \quad r$ 


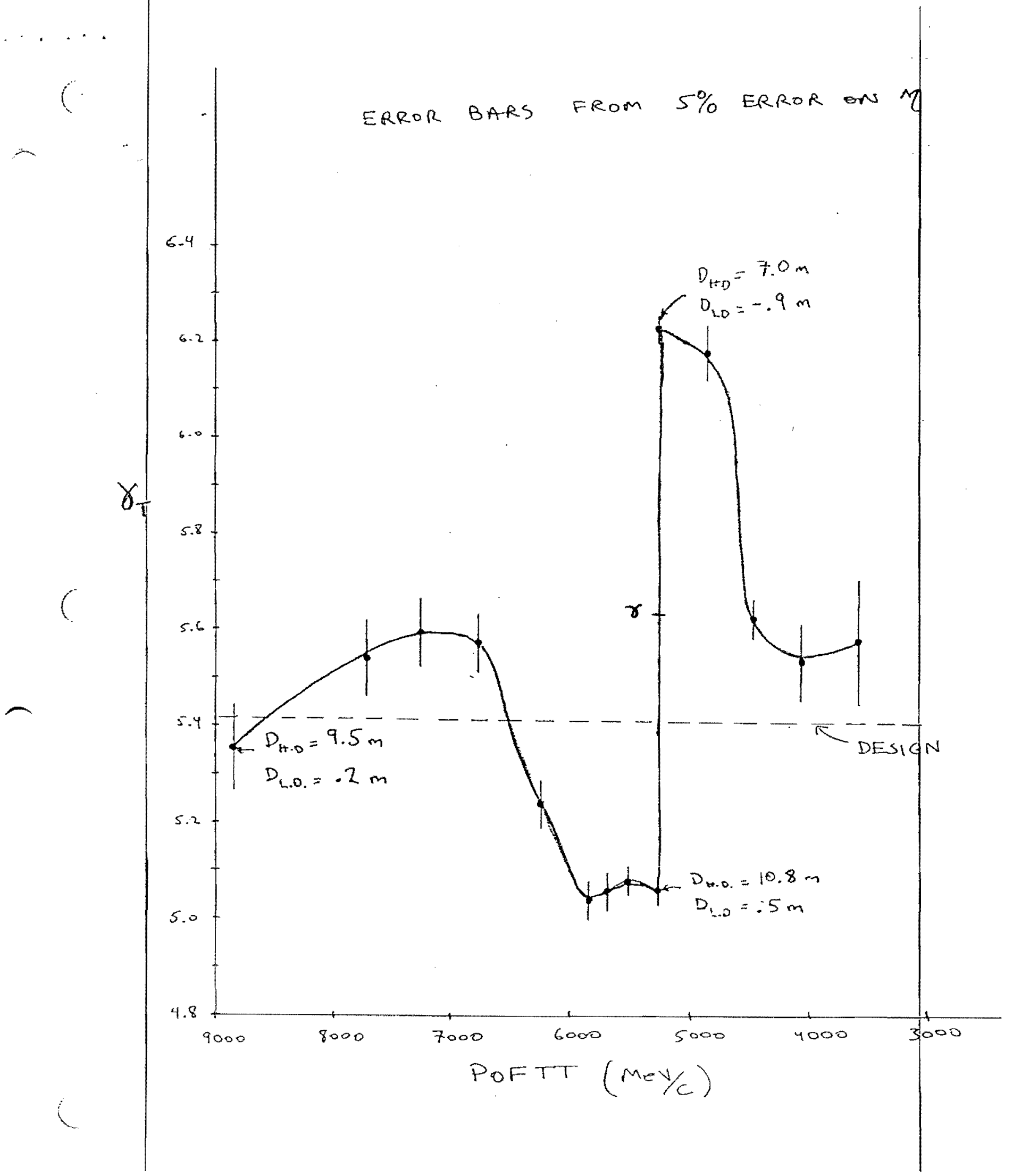

FIG. 2: Transition gamma as a function of beam energy; also shown are the dispersions at the straight sections. 


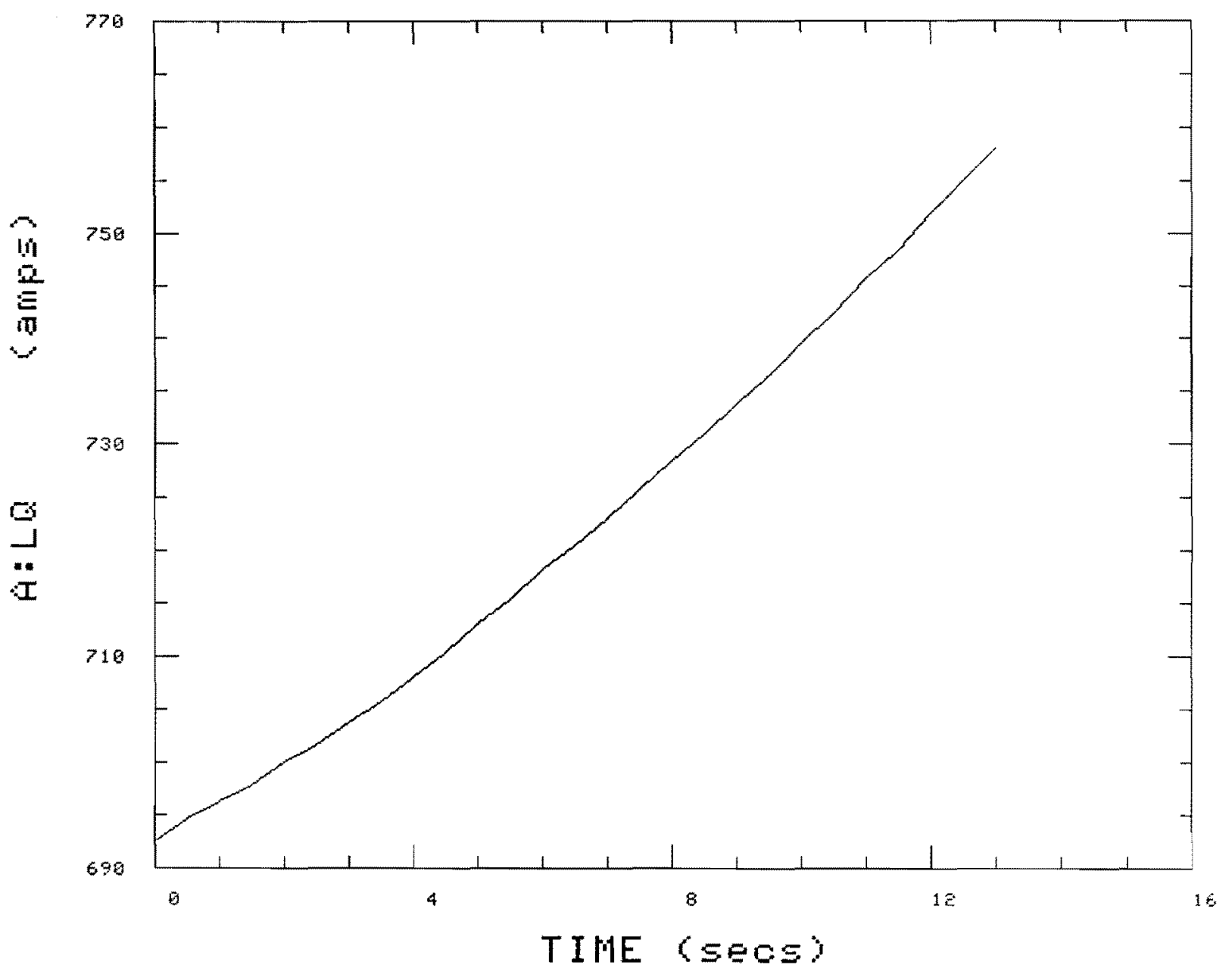

FIG. 3: LQ current settings as a function of time during transition crossing ramp 


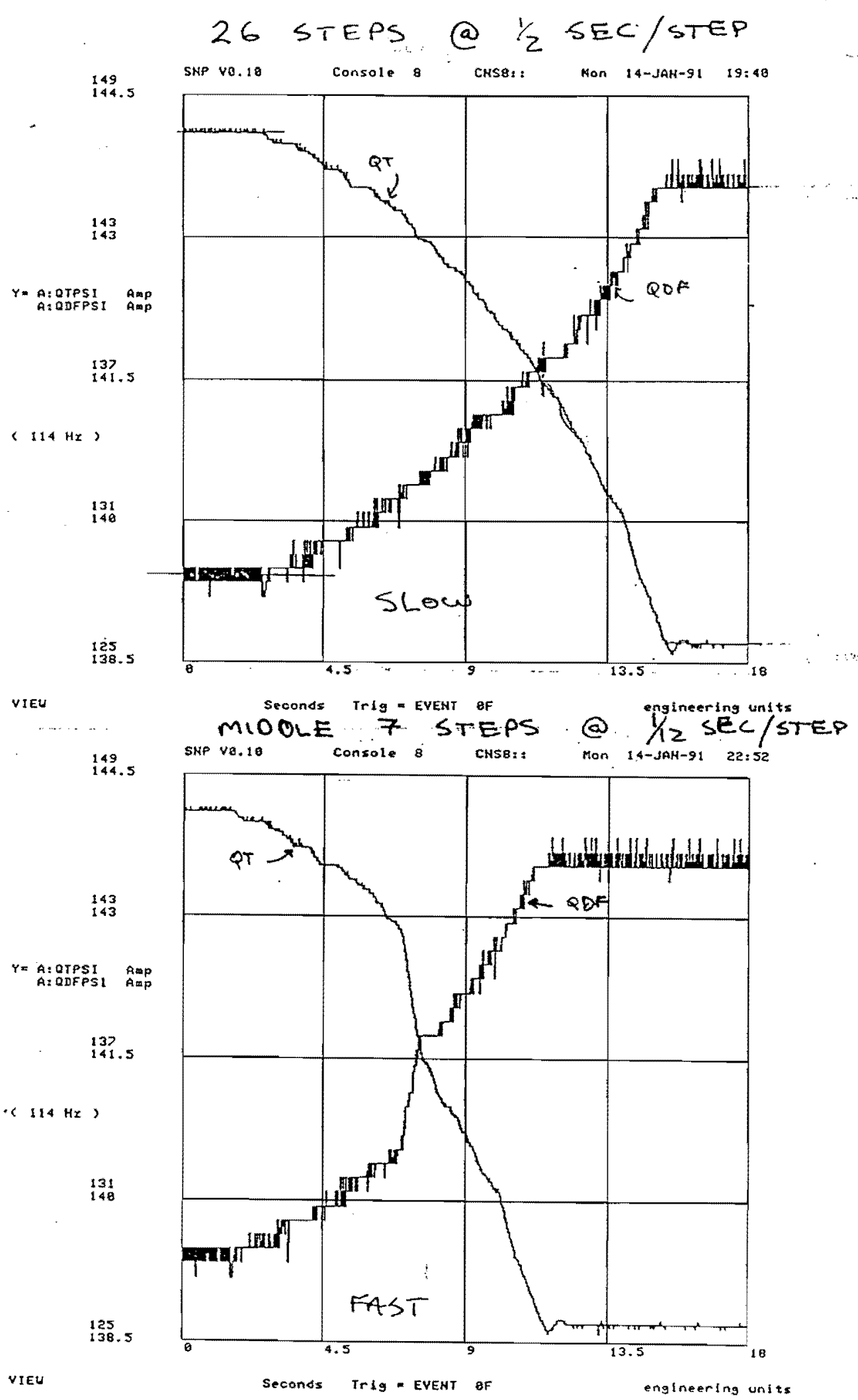

FIG. 4: Top plot shows QT and QDF current readback as a function of time during transition crossing for the slow ramp (1/2 sec per step); Bottom plot shows same but for fast ramp (steps 12-18 6 times faster) 


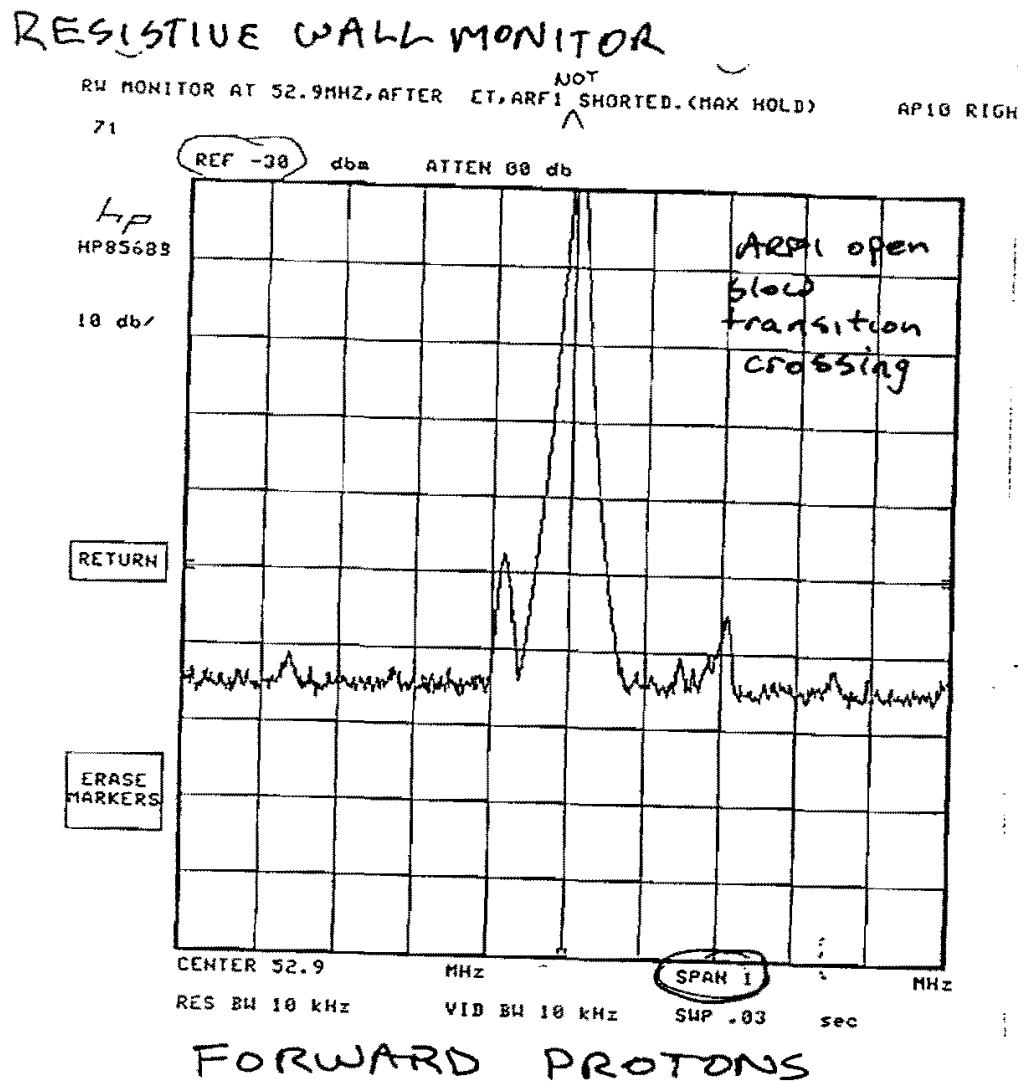

RH MONITOR, AFTER FASTER ET CROSSIHE, 5. SHA, ARF 1 SHORTEO. APIB RI 85

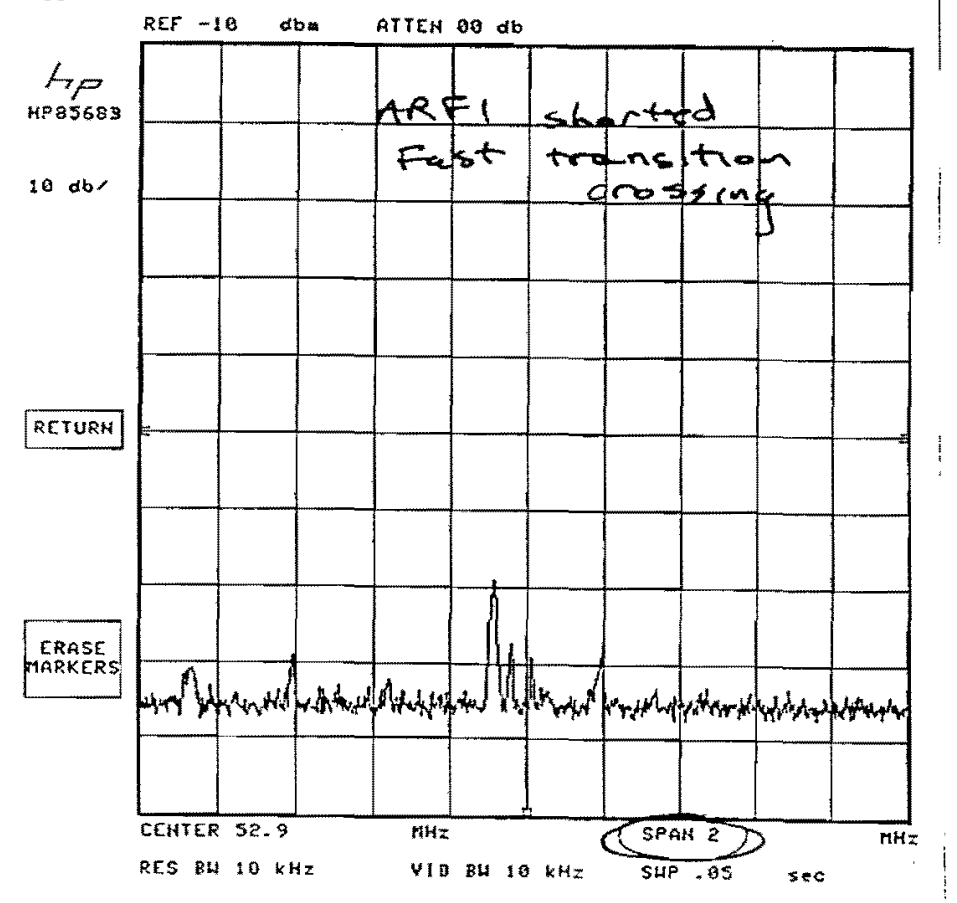

FIG. 5: "Maximum hold" frequency spectrum from resistive wall monitor during transition crossing; top plot is with no improvements and bottom plot is with ARF1 shorted and faster ramp 
त्ञ

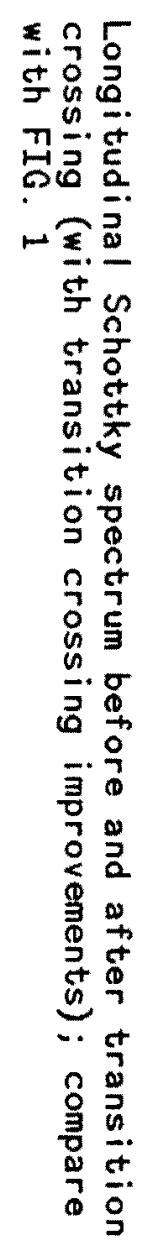

LONGITUDINAL SCHOTTHY,

before and ofter trainsition, both gaps shorted, fast ramp Ref LUI -20 dB

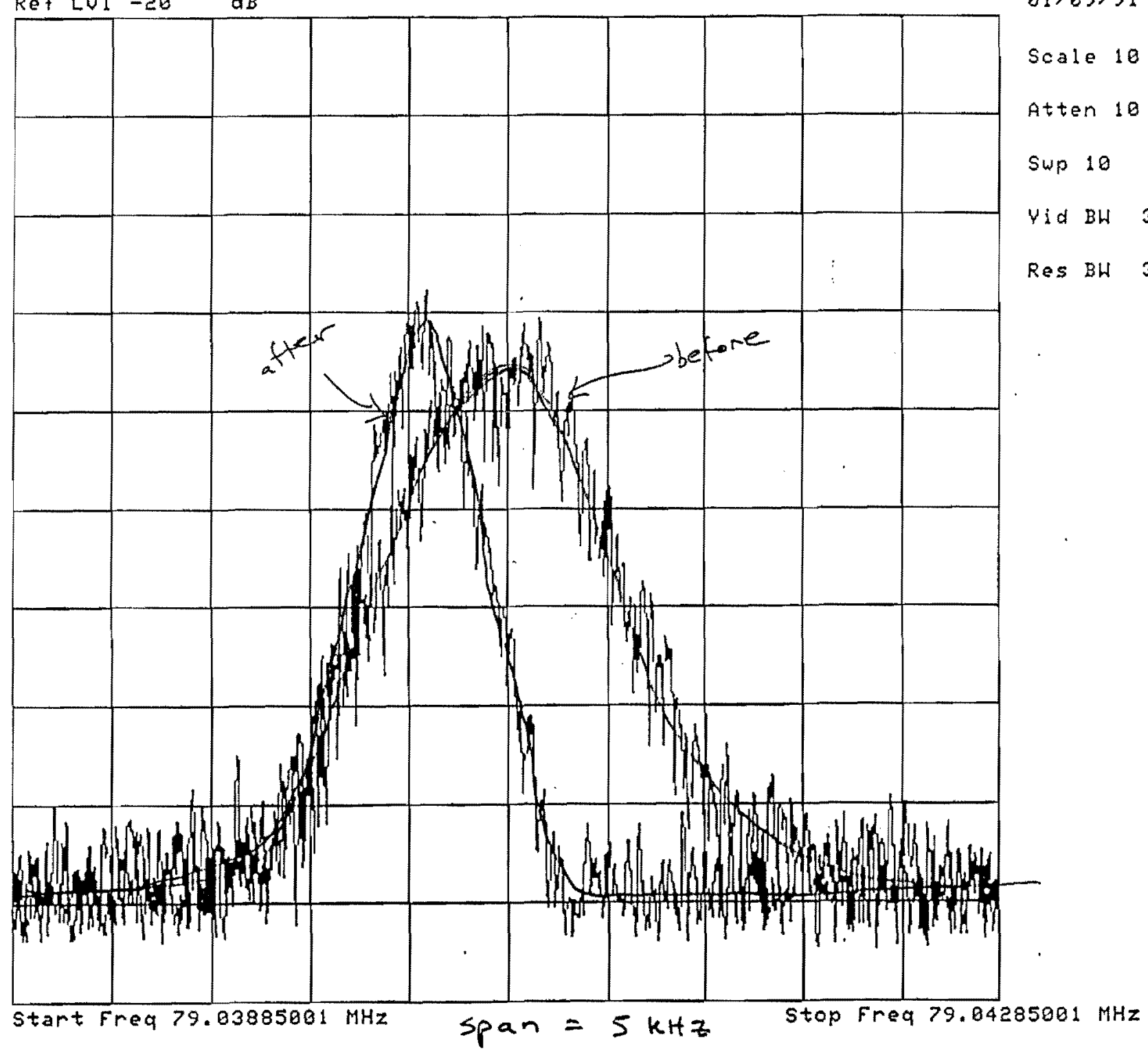

$7 M A \bar{P}$

1607 sec $\mathrm{Hz}$ 3e $\mathrm{Hz}$ 